\title{
Homocysteine Induced Cerebrovascular Dysfunction: A Link to Alzheimer's Disease Etiology
}

\author{
P.K. Kamat, J.C. Vacek, A. Kalani and N. Tyagi ${ }^{*}$
}

Department of Physiology and Biophysics, School of Medicine, University of Louisville, and Louisville, KY 40202, USA

\begin{abstract}
A high serum level of homocysteine, known as hyperhomocystenemia (HHcy) is associated with vascular dysfunction such as altered angiogenesis and increased membrane permeability. Epidemiological studies have found associations between HHcy and Alzheimer's disease (AD) progression that eventually leads to vascular dementia (VaD). $\mathrm{VaD}$ is the second most common cause of dementia in people older than 65 , the first being $\mathrm{AD}$. $\mathrm{VaD}$ affects the quality of life for those suffering by drastically decreasing their cognitive function. $\mathrm{VaD}$, a cerebrovascular disease, generally occurs due to cerebral ischemic events from either decreased perfusion or hemorrhagic lesions. HHcy is associated with the hallmarks of dementia such as tau phosphorylation, A $\beta$ aggregation, neurofibrillary tangle (NFT) formation, neuroinflammation, and neurodegeneration. Previous reports also suggest HHcy may promote AD like pathology by more than one mechanism, including cerebral microangiopathy, endothelial dysfunction, oxidative stress, neurotoxicity and apoptosis. Despite the corelations presented above, the question still exists - does homocysteine have a causal connection to $\mathrm{AD}$ ? In this review, we highlight the role of HHcy in relation to $\mathrm{AD}$ by discussing its neurovascular effects and amelioration with dietary supplements. Moreover, we consider the studies using animal models to unravel the connection of Hcy to AD.
\end{abstract}

Keywords: Alzheimer's disease, blood brain barrier, cerebrovascular pathology, homocysteine, vascular dementia,

\section{INTRODUCTION}

Homocysteine (Hcy), a key metabolic intermediate in sulfur-containing amino acid metabolism [1] is an independent risk factor for cerebrovascular disease [2]. Hcy is an excitatory amino acid, which markedly enhances the vulnerability of neurons to oxidative injury [3]. An elevated plasma level of Hcy $(>14 \mu \mathrm{M})$ is termed as hyperhomocysteinemia (HHcy), which has emerged as an independent risk factor for several neurodegenerative diseases such as Alzheimer's disease (AD) [4], stroke [5] and vascular dementia [6]. In recent years, it has become clear that elevated levels of Hcy elicits neuronal death in a variety of neuronal cell types, including hippocampal and cortical neurons [7], cerebellar granule cells [8], and SH-SY5Y cells [9]. One study has shown an association with Hcy and hippocampal plasticity and synaptic transmission resulting in learning and memory deficits [10]. Recently, it has been suggested that intracerebral administration of Hcy affected both long- and short-term memory [3]. One explanation for the mechanism of Hcy induced neurotoxicity is the autooxidation of Hcy leading to cellular oxidative stress through the formation of reactive oxygen species, which causes neuroinflammation and apoptosis [11].

*Address correspondence to this author at the Department of Physiology and Biophysics, Health Sciences Center, A-1201, University of Louisville, Louisville, KY 40202; Tel: 502-852-4145; Fax: 502-852-6239;

E-mail:n0tyag01@louisville.edu

\section{METABOLISM OF HOMOCYSTEINE}

Methylenetetrahydrofolate reductase (MTHFR), a key enzyme in the metabolism of folate and homocysteine, catalyzes the synthesis of the main circulatory form of folate, 5 -methyltetrahydrofolate (5-methylTHF). 5-MethylTHF is used for remethylation of homocysteine to methionine and Sadenosyl methionine (SAM), a universal methyl donor [12]. In the liver and kidneys, the choline metabolite betaine can serve as an alternate methyl donor for homocysteine remethylation, particularly when folate-dependent remethylation is disturbed [13]. Homocystinuria, an inborn error of metabolism, can be caused by different genetic deficiencies, including severe MTHFR deficiency. MTHFR mutations in homocystinuric patients are associated with low levels of enzyme activity (0-20\%), marked HHcy and decreased levels of serum folic acid, methionine and SAM [14]. Clinical features vary but often include developmental delays, mental retardation, motor abnormalities, psychiatric disorders, cerebral atrophy, demyelination, and thrombosis [15]. Several studies observed high plasma Hcy in patients with mild cognitive impairment, $\mathrm{AD}$, depression and schizophrenia suggesting the association between HHcy and association with HHcy and neurological pathology [16].

HHcy has been associated with B vitamin deficiencies of folate, B6, and B12 due to their role as cofactors in the metabolism of Hcy. Vitamin B12 is also a cofactor in other methylation reactions. In particular, vitamin B12 deficiency has been shown to result in cognitive impairment [17]. It is not clear whether the cognitive impairment is a direct consequence of B12 deficiency or whether it is a result of the 


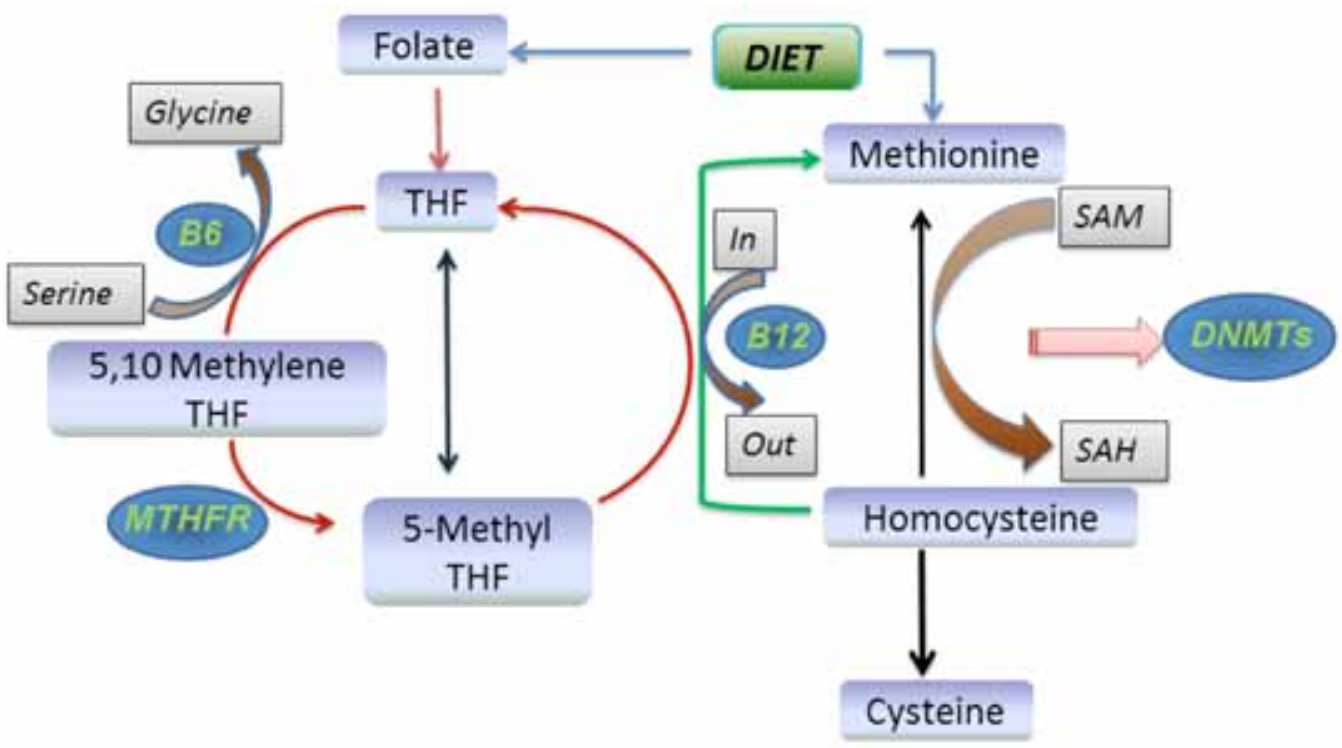

Fig. (1). Homocysteine-Folate Pathway: The metabolic interactions between folate, methionine and homocysteine are shown here. Folate from the diet is absorbed and converted to methylenetetrahydrofolate (MTHF) with the formation of high-energy phosphate bonds by the action of MTHF reductase (MTHFR), methyl tetrahydrofolate is yielded which methylates homocysteine to methionine. Methionine is converted to SAM, the universal methyl donor. SAM provides methyl groups for several nucleic acid methylation reactions and gets reduced to S-adenosylhomocysteine which then reforms homocysteine and reinitiates the cycle. Abbreviations: SAM; S-adenosylmethionine, SAH; S-adenosylhomocysteine, MTHFR; methylenetetrahydrofolate reductase; B6 and B12; Vitamin B6 and Vitamin 12 respectively.

subsequent HHcy. One such mechanism for this observation could be linked to myelin basic protein. Myelin basic protein is methylated on an arginine group and a defect in methylation could produce an unstable protein, leading to neurological disorders [18].

As mentioned above folic acid is a B-group water-soluble vitamin. It is comprised of the aromatic pterydine ring linked to para-aminobenzoic acid and one or more glutamate residues. Folate derivatives are important vitamins that transfer one-carbon units for several critical cellular reactions. The in folate cycle in association with Hcy is depicted in Fig. (1). Neuronal function relies heavily on the supply of methyl groups for such functions as neurotransmitter synthesis and membrane lipid metabolism. Furthermore, folates are required for the metabolism of Hcy. The regulation of Hcy allows for the maintenance of neuroplasticity and neuronal integrity, when HHcy develops neurotoxicity is known to occur [19]. Such detrimental effects can be attributed to mechanisms such as excitotoxicity and mitochondrial dysfunction, which may lead to apoptosis [20]. One study has shown HHcy in mice along with B-vitamin deficiency diet cause cerebral microvascular rarefaction which may bring about cognitive dysfunction. Clinical reports suggest that disease progression of a dementia patient with HHcy and associated morbidities can be lowered by supplementation of food or nutrient enriched with B vitamins. Another study has shown the food supplementation of folates and vitamin B12 ameliorating HHcy and thus Hcy induced tau hyperphos-phorylation as well as protein phosphatase-2A (PP2A) inactivation [21]. After reviewing the literature it is apparent that the association with HHcy and cognitive impairment is evident and should be elucidated further.

\section{OXIDATIVE STRESS AND NEUROINFLAMMATION}

Experimental and clinical studies have shown numerous inflammatory mediators being implicated in neurological disorders. Histological analysis of human brain tissue from individuals with $\mathrm{AD}$ strongly suggests the existence of a chronic inflammatory state in brain tissue leading to neuronal loss [22]. Other studies confirm the association of oxidative stress, HHcy, altered cerebrovascular remodeling and neuroinflammation with AD [3, 23]. HHcy has been documented to show changes in the structure and function of cerebral blood vessels via oxidative stress, which plays a key role in cerebrovascular endothelial dysfunction [24]. Oxidative stress and endothelial dysfunction led to structural deformities in the cerebral blood vessels that can impair perfusion with subsequent neuronal dysfunction and recognized as an important contributing factor in the pathogenesis of $\mathrm{AD}$ and $\mathrm{VaD}$. One novel study has shown intracerebroventricular injections of $\mathrm{Hcy}$ in rats inducing lipid peroxidation, increased malondialdehyde and superoxide anion levels in brain tissue leading to impaired memory retention in the passive avoidance learning test [25]. It is apparent that Hcy injections in rodent brains led to severe oxidative stress, neuroinflammation and cognitive impairment $[3,10]$.

\section{MMPS AND BLOOD BRAIN BARRIER (BBB) DAMAGE}

HHcy causes increased expression of matrix metalloproteinases (MMPs) [26]. Oxidative stress has been suggested to be involved in neurovascular dysfunction in part by activation of MMPs along with apoptosis induced by 
Hcy [3]. One study analyzing this effect used a heterozygous cystathionine-b-synthase knockout mouse model; the resulting HHcy showed increased brain permeability in relation to increased MMP-9 and MMP-2 activity and suppression of tissue inhibitors of metalloproteinase (TIMPs) [27]. These effects led to the degradation of extracellular matrix (ECM) and disruption of the BBB. In mild HHcy, increased permeability of the $\mathrm{BBB}$ precedes the onset of cerebral pathology related to the progression of $\mathrm{AD}$ [28]. MMPs are membrane-bound, zinc-binding proteolytic enzymes and activated by other proteases and free radicals in the ECM. The MMPs are essential for the breakdown of ECM components of cerebral blood vessels and neurons [29]. In general, MMPs are released as a zymogen and activated by proteolytic cleavage of the $\mathrm{N}$-terminal domain. MMP-9 is usually low and its expression can be induced by various pro-inflammatory factors such as cytokines. MMP-2 is constitutively expressed in several cell types and rarely inducible. In the central nervous system (CNS) MMPs, especially MMP-9, are implicated in the pathogenesis of several CNS diseases such as stroke, AD and multiple sclerosis [30]. Several pro-inflammatory factors including cytokines, endotoxins, and oxidative stress have been shown to up-regulate MMP-9 in astrocytes in vitro [31]. The accumulation of toxic free radicals plays an essential role in BBB disruption through MMP activation [32]. MMPs, in particular MMP-2 and MMP-9, are heavily implicated in the pathogenesis of hemorrhagic events; leaky vessels and BBB damage [33]. These events escalate to clinically significant neurologic disease.

\section{ENDOTHELIAL DYSFUNCTION}

Endothelial cells are key modulators of inflammation and angiogenesis [34]. Several studies confirm endothelial dysfunction may be partly caused by oxidation related to the effects of raised total plasma homocysteine [35]. Endothelial dysfunction is also increasingly implicated in the development of neurodegenerative diseases such as AD [36]. Cell adhesion molecules play an important role in inflammatory responses in brain endothelial cells. The vascular cell adhesion molecule (VCAM-1) and intercellular adhesion molecule 1 (ICAM-1), one of the inducible immunoglobulins expressed on several cell types, play an important role in a number of inflammatory and immune responses [37]. Upregulation of these adhesion molecules in astrocytes is required for monocyte-astrocyte interaction, leading to an increased infiltration of circulating monocytes into the CNS observed in patients with AD. Endothelial cells in the brain regulate the neuronal milieu both by their synthetic functions as well as by their BBB regulation. However, chronic inflammation through circulating monocytes and cytokines are tightly linked to diseases associated with endothelial dysfunction. Therefore, disturbances in endothelial function could result in a noxious neuronal environment facilitating AD pathogenesis.

\section{BBB DYSFUNCTION AND AD PATHOLOGY}

The BBB is formed by highly specialized endothelial cells that line brain capillaries and allow specific molecular exchange between micro-vessels, microglia and neurons. The BBB relies on the tight junctions present between endothelial cells of the capillaries to provide a relatively closed environment for the brain. Alteration of tight junction proteins promotes BBB dysfunction, which is associated with a reduction of cerebral blood flow, hypoxia and accumulation of neurotoxic molecules such as free radicals and inflammatory molecules in the brain [38]. In contrast to leaky vessels in visceral organs, the $\mathrm{BBB}$ restricts entry of polar molecules into the brain but at the same time allows limited nutrients such as vitamins, glucose, and amino acids through specific transporters [39]. BBB disruption promotes micro-vascular degeneration, accumulation of toxic substances (Beta amyloid, NFT and Tau hyperphosphorylation), neurodegeneration, neuroinflammation and memory impairment [40].

\section{HOMOCYSTEINE AND VASCULAR DEMENTIA}

Vascular dementia $(\mathrm{VaD})$ is defined as the loss of cognitive function resulting from cerebrovascular disease that damages the hippocampus and other cognitive centers, which important for memory, cognition, and behavior [41]. It is the second most common form of dementia after AD, accounting for $20 \%$ of all dementia cases worldwide [42]. In addition, $\mathrm{VaD}$ is thought to occur in $20 \%$ to $55 \%$ of $\mathrm{AD}$ cases [43]. Previous studies, which have induced HHcy in rodents, produced cognitive decline, systemic vascular inflammation, atherosclerosis and a loss of central cholinergic neurons [44]. $\mathrm{AD}$ and $\mathrm{VaD}$ share many risk factors, one such being HHcy, thus suggesting related etiology and pathogenesis [45]. It is common for patients of each disease to share similar symptoms [46]. Therefore, the differentiation from $\mathrm{AD}$ to $\mathrm{VaD}$ is based on evidence of cerebrovascular function alteration and pathology in $\mathrm{VaD}$ (Fig. 2). Furthermore, it has been suggested that dementia prevention can be effective without delay if the vascular components are aggressively targeted through the treatment of vascular risk factors such as HHcy [47].

\section{HOMOCYSTEINE AND ALZHEIMER'S DISEASE}

Alzheimer's disease (AD) is characterized by cerebrovascular damage and neuronal atrophy leading to a progressive cognitive decline which is also known as $\mathrm{VaD}$ $[48,49]$. AD shares many risk factors with cerebrovascular diseases, thus hinting at a potential vascular etiology [50, 51]. In addition, numerous epidemiological studies have observed micromolar increases in Hcy to be associated with increased risk of stroke, white matter disease, and cognitive dysfunctions that range in severity from mild cognitive impairment to AD [52]. The hallmark histologic findings for $\mathrm{AD}$ include beta-amyloid $(\mathrm{A} \beta)$ plaques and angiopathy, NFTs containing hyperphosphorylated tau proteins [53], $\mathrm{BBB}$ leakage, and increased microglial reactivity [23]. One hypothesis suggests the accumulation of $A \beta$ is the most important cascade for the development of AD. A $\beta$-peptides (40, 42 or 43 amino acids) originate from the amyloid precursor protein (APP) by cleavage with $\alpha-, \beta$ - and $\gamma$ secretases during sudden $A \beta$-production. Clearance of the peptides may trigger neurodegeneration [54]. In AD 


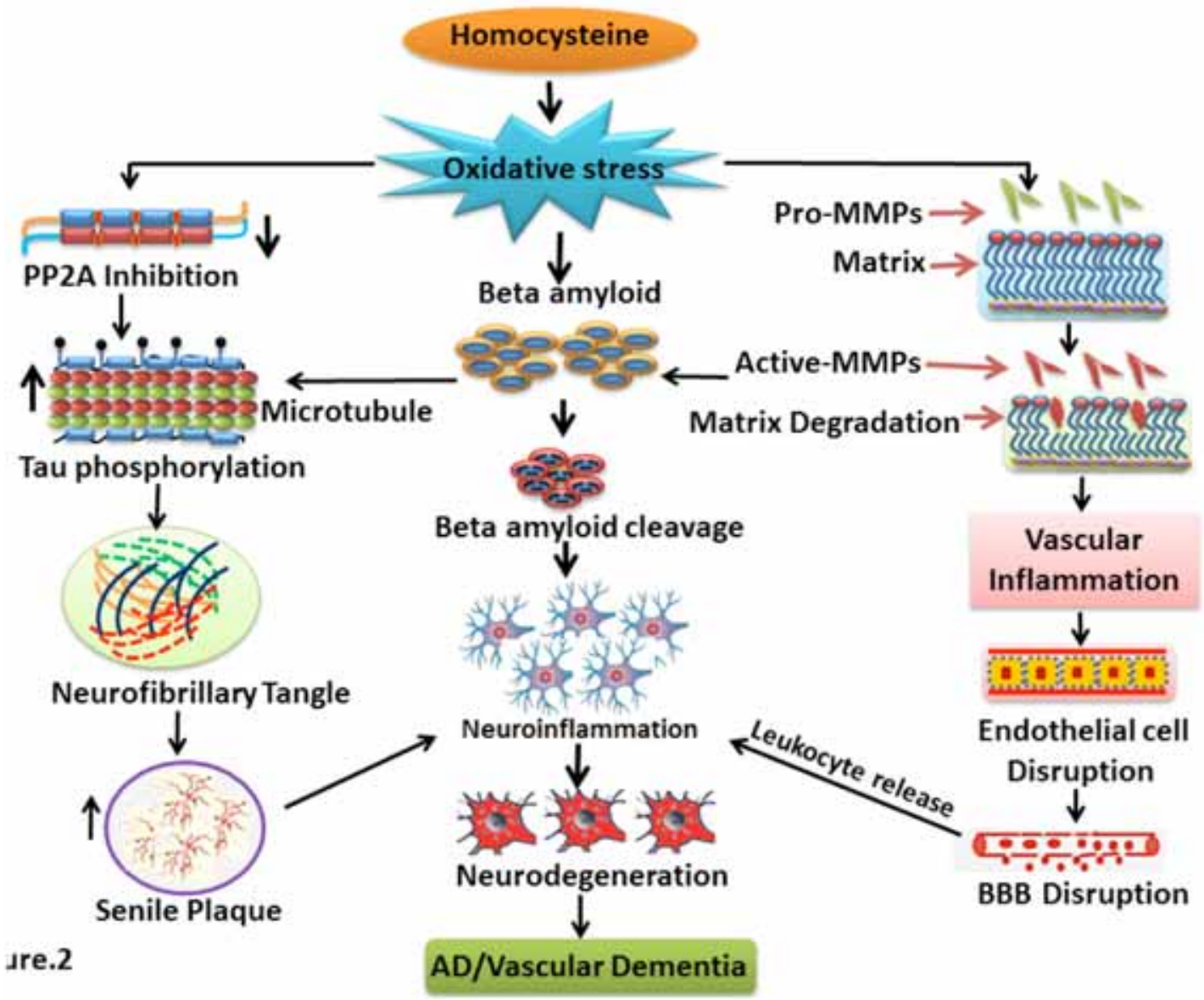

Fig. (2). Homocysteine and Vascular Dementia: Flow diagram showing the mechanism of homocysteine induced vascular dementia. Homocysteine produces oxidative stress in the initial stage. Further oxidative stress leads to activation of MMPs as well as PP2A inhibition. Activated MMPs promote matrix degradation, vascular inflammation, endothelial dysfunction and finally BBB dysfunction. Activated MMPs may also promote beta amyloid $(\mathrm{A} \beta)$ cleavage and neuroinflammation. PP2A inhibition by homocysteine causes Tau hyperphosphorylation. These events result in the neurofibrillary and senile plaque formation in the brain. Further, senile plaque and neurofibrillary tangle formation promotes neurodegeneration and thus initiates AD and vascular dementia like symptoms.

progression Hcy over long periods may cause dysfunctional $\mathrm{A} \beta$-clearance as well as BBB impairment which may initiate cerebrovascular dysfunction or inflammatory processes leading to the development of AD [46].

\section{BETA AMYLOID (Aß) AND TAU HYPERPHOS- PHORYLATION}

$\mathrm{A} \beta$ plaques and Tau hyperphosphorylation are characteristic features of AD associated with the deterioration of cognitive functions [55]. Neurofibrillary tangles, senile plaques, and synaptic loss in the affected brain regions such as cortex and hippocampus are other typical features of AD [56]. A potential mechanistic association of these features could be traced to HHcy. One study concluded HHcy increases levels of $A \beta$ transporters in micro-vessels that form the $B B B$, elevates $A \beta$ content $(A \beta 40$ and $A \beta 42)$, and impairs cognitive function [57]. Chai et al. [58] demonstrated the elevation of plasma Hcy could induce $A \beta$ peptide accumulation and increase AD-like tau hyperphos- phorylation in rats [59]. In addition to this Hcy sensitizes the neurons to $A \beta$ toxicity and impairs DNA repair in hippocampal neurons [60]. Another study with folate and vitamin-B12 treatment in rodents showed decrease $A \beta$ production and attenuation of memory impairment induced by HHcy [58] Furthermore, Zhang et al. [59] reported homocysteine injections in intracerebroventricular regions rat brain which dispersed into whole brain led to increased levels of amyloid precursor protein (APP) levels. The associations of HHcy on AD features in animal models with a normal gene background are not currently elucidated and make for a novel ground of research. New efforts using these models could give clues not only to $\mathrm{AD}$ but also $\mathrm{VaD}$ and other dementia subtypes.

\section{ANIMAL MODEL OF HHCY SHOW POTENTIAL LINK TO AD}

Several animal models have shown a role of HHcy in cerebrovascular pathology, cognitive decline and learning disabilities [44]. Koladiya et al. [61] reported that HHcy 
induced via administration of L-methionine in rodents has been reported to produce a significant degree of $\mathrm{VaD}$. Other studies have shown intracerebral Hcy injections in rodent brains to produce $\mathrm{AD}$ like symptoms [3,10]. Another project has shown a transgenic mouse model such as CBS (Cystathione- $\beta$ synthase) knockout mice inducing HHcy leading to $\mathrm{A} \beta$ toxicity [62]. It is important to note that a reported linking of $\mathrm{Hcy}$ to $A \beta$ induced hippocampal neurotoxicity has been observed, a potential source of $\mathrm{AD}$ [60].

\section{CONCLUSION}

Although AD and many other dementia subtypes are not believed to be primarily vascular diseases, it is important to reiterate many of the subtypes share common risk factors with vascular diseases. As discussed above, many studies have shown dysfunctional vessels across the subtypes of cognitive disorders. One known condition to affect the cerebrovascular homeostasis is HHcy and evidence suggests HHcy may promote dementia by more than one mechanism, including cerebral microangiopathy, endothelial dysfunction, oxidative stress, neuronal damage, enhancement of betaamyloid peptide-mediated vascular toxicity, neurotoxicity, and apoptosis. Therefore, further studies particularly with animal models make for promising returns as to the etiology of dementia disorders. Furthermore, because effective treatments for HHcy have been studied and supported, potential novel therapies for patients with dementia could be devised and clinically tested.

\section{ABBREVIATIONS}

$\begin{array}{ll}\mathrm{Hcy} & =\text { homocysteine, } \\ \mathrm{HHcy} & =\text { hyperhomocysteinemia, } \\ \mathrm{AD} & =\text { Alzheimer's disease, } \\ \mathrm{VaD} & =\text { vascular dementia, } \\ \mathrm{A} \beta & =\text { Beta amyloid, } \\ \mathrm{ROS} & =\text { reactive oxygen species, } \\ \mathrm{BBB} & =\text { blood brain barrier, } \\ \mathrm{MMPs} & =\text { matrix metalloproteinases, } \\ \mathrm{MTHFR} & =\text { methylenetetrahydrofolate reductase, } \\ \mathrm{SAM} & =\text { S-adenosylmethionine, } \\ (\mathrm{TIMPs}) & =\text { tissue inhibitor of metalloproteinases } \\ (\mathrm{ECM}) & =\text { extracellular matrix } \\ (\mathrm{CNS}) & =\text { central nervous system }\end{array}$

\section{CONFLICT OF INTEREST}

The authors confirm that this article content has no conflict of interest.

\section{ACKNOWLEDGEMENTS}

This work is supported in part by the NIH grant HL107640 to NT.

\section{REFERENCES}

[1] Prudova A, Bauman Z, Braun A, Vitvitsky V, Lu SC, Banerjee R. S-adenosylmethionine stabilizes cystathionine beta-synthase and modulates redox capacity. Proc Natl Acad Sci USA 2006; 103: 6489-94.

[2] Garcia A, Zanibbi K. Homocysteine and cognitive function in elderly people. Cmaj 2004; 171: 897-904.

[3] Kamat PK, Kalani A, Givvimani S, Sathnur PB, Tyagi SC, Tyagi N. Hydrogen sulfide attenuates neurodegeneration and neurovascular dysfunction induced by intracerebral-administered homocysteine in mice. Neuroscience 2013; 252: 302-19.

[4] Zhuo JM, Wang H, Pratico D. Is hyperhomocysteinemia an Alzheimer's disease (AD) risk factor, an AD marker, or neither? Trends Pharmacol Sci 2011; 32: 562-71.

[5] Brustolin S, Giugliani R, Felix TM. Genetics of homocysteine metabolism and associated disorders. Braz J Med Biol Res 2010; 43:1-7.

[6] Nilsson K, Gustafson L, Hultberg B. Plasma homocysteine and cognition in elderly patients with dementia or other psychogeriatric diseases. Dement Geriatr Cogn Disord 2010; 30: 198-204.

[7] Ho V, Massey TE, King WD. Influence of thymidylate synthase gene polymorphisms on total plasma homocysteine concentrations. Mol Genet Metab 2010; 101: 18-24.

[8] Kuszczyk M, Gordon-Krajcer W, Lazarewicz JW. Homocysteineinduced acute excitotoxicity in cerebellar granule cells in vitro is accompanied by PP2A-mediated dephosphorylation of tau. Neurochem Int 2009; 55: 174-80.

[9] Park YJ, Jang Y, Kwon YH. Protective effect of isoflavones against homocysteine-mediated neuronal degeneration in $\mathrm{SH}$ SY5Y cells. Amino Acids 2010; 39: 785-94.

[10] Ataie A, Sabetkasaei M, Haghparast A, Moghaddam AH Kazeminejad B Neuroprotective effects of the polyphenolic antioxidant agent, Curcumin, against homocysteine-induced cognitive impairment and oxidative stress in the rat. Pharmacol Biochem Behav 2010; 96: 378-85.

[11] Yan SK, Chang T, Wang H, Wu L, Wang R, Meng QH. Effects of hydrogen sulfide on homocysteine-induced oxidative stress in vascular smooth muscle cells. Biochem Biophys Res Commun 2006; 351: 485-91.

[12] Chiang PK, Gordon RK, Tal J, Zeng GC, Doctor BP, Pardhasaradhi K, McCann PP. S-Adenosylmethionine and methylation. Faseb J 1996; 10: 471-80.

[13] Caudill MA, Dellschaft N, Solis C, et al. Choline intake, plasma riboflavin, and the phosphatidyletha-nolamine N-methyltransferase G5465A genotype predict plasma homocysteine in folate-deplete Mexican-American men with the methylenetetrahydrofolate reductase 677TT genotype. J Nutr 2009; 139: 727-33.

[14] Aisen PS, Schneider LS, Sano M, et al. High-dose B vitamin supplementation and cognitive decline in Alzheimer disease: a randomized controlled trial. JAMA 2008; 300: 1774-83.

[15] Chen J, Zhang I, Cheng L, Li Y. The effect of polymorphisms of MTHER gene and vitamin B on hyperhomocysteinemia. J Tongji Med Univ 2001; 21: 17-20.

[16] Folstein M, Liu T, Peter I, et al. The homocysteine hypothesis of depression. Am J Psychiatry 2007; 164: 861-67.

[17] Moore E, Mander A, Ames D, Carne R, Sanders K, Watters D Cognitive impairment and vitamin B12: a review. Int Psychogeriatr 2012; 24: 541-56.

[18] Sponne IE1, Gaire D, Stabler SP, et al. Inhibition of vitamin B12 metabolism by $\mathrm{OH}$-cobalamin c-lactam in rat oligodendrocytes in culture: a model for studying neuropathy due to vitamin B12 deficiency. Neurosci Lett 2000; 3: 191-4.

[19] Kruman, II, Mouton PR, Emokpae R, Jr., Cutler RG, Mattson MP. Folate deficiency inhibits proliferation of adult hippocampal progenitors. Neuroreport 2000; 16: 1055-59. 
[20] Kronenberg G, Colla M, Endres M. Folic acid, neurodegenerative and neuropsychiatric disease. Curr Mol Med 2009; 9: 315-23.

[21] Zhang CE, Tian Q, Wei W, et al. Homocysteine induces tau phosphorylation by inactivating protein phosphatase $2 \mathrm{~A}$ in rat hippocampus. Neurobiol Aging 2008; 29: 1654-65.

[22] Vezzani A. Inflammation and epilepsy. Epilepsy Curr 2005; 5:1-6.

[23] Kalani A, Kamat PK, Givvimani S, et al. Nutri-epigenetics ameliorates blood-brain barrier damage and neurodegeneration in hyperhomocysteinemia: role of folic acid. J Mol Neurosci 2014; 2 : 202-15.

[24] Rivera J, Sobey CG, Walduck AK, Drummond GR. Nox isoforms in vascular pathophysiology: insights from transgenic and knockout mouse models. Redox Rep 2010; 15: 50-63.

[25] Troen AM. The central nervous system in animal models of hyperhomocysteinemia. Prog Neuropsychopharmacol Biol Psychiatry 2005; 29: 1140-51

[26] Kalani A, Kamat PK, Familtseva A, et al. Role of microRNA29b in blood-brain barrier dysfunction during hyperhomocysteinemia: an epigenetic mechanism. J Cereb Blood Flow Metab. 2014; 7: 121222.

[27] Tyagi N, Qipshidze N, Munjal C, et al. Tetrahydrocurcumin ameliorates homocysteinylated cytochrome-c mediated autophagy in hyperhomocysteinemia mice after cerebral ischemia. J Mol Neurosci 2012; 47: 128-38.

[28] Rhodehouse BC1, Mayo JN, Beard RS Jr, Chen CH, Bearden SE. Opening of the blood-brain barrier before cerebral pathology in mild hyperhomocysteinemia. PLoS One 2013; 5: e63951.

[29] Peng H, Cheng H, Xing HY, Li G, Yu FQ. Effect mechanism of Hcy on damage of BBB and the intervention of the compounded vitamin in rat. Zhonghua Yi Xue Za Zhi 2010; 90: 1642-44.

[30] Yong VW, Power C, Forsyth P, Edwards DR. Metalloproteinases in biology and pathology of the nervous system. Nat Rev Neurosci 2001; 2: 502-11.

[31] Lee WJ, Shin CY, Yoo BK, et al. Induction of matrix metalloproteinase-9 (MMP-9) in lipopolysaccharide-stimulated primary astrocytes is mediated by extracellular signal-regulated protein kinase 1/2 (Erk1/2). Glia 2003; 41: 15-24.

[32] Klein T, Bischoff R. Physiology and pathophysiology of matrix metalloproteases. Amino Acids 2011; 41: 271-90.

[33] Dal-Pizzol F, Rojas HA, dos Santos EM, et al. Matrix metalloproteinase-2 and metalloproteinase-9 activities are associated with blood-brain barrier dysfunction in an animal model of severe sepsis. Mol Neurobiol 2013; 48: 62-70.

[34] De Caterina R, Massaro M, Scoditti E, Annunziata Carluccio M Pharmacological modulation of vascular inflammation in atherothrombosis. Ann N Y Acad Sci 2010; 1207: 23-31.

[35] Abdulle AM, Pathan JY, Moussa N, Gariballa S. Association between homocysteine and endothelial dysfunction markers in stroke disease. Nutr Neurosci 2010; 13: 2-6.

[36] Salmina AB, Inzhutova AI, Malinovskaya NA, Petrova MM. Endothelial dysfunction and repair in Alzheimer-type neurodegeneration: neuronal and glial control. J Alzheimers Dis 2010; $22: 17-36$

[37] Cook-Mills JM. VCAM-1 signals during lymphocyte migration: role of reactive oxygen species. Mol Immunol 2002; 39: 499-08.

[38] Kalaria RN. Vascular basis for brain degeneration: faltering controls and risk factors for dementia. Nutr Rev 2010; 68 Suppl 2: S74-87.

[39] Zlokovic BV. Neurovascular pathways to neurodegeneration in Alzheimer's disease and other disorders. Nat Rev Neurosci 2011; 12: 723-38.

[40] Bell RD. The imbalance of vascular molecules in Alzheimer's disease. J Alzheimers Dis 2012; 32: 699-09.

[41] Quadri P, Fragiacomo C, Pezzati R, et al. Homocysteine, folate, and vitamin B-12 in mild cognitive impairment, Alzheimer disease, and vascular dementia. Am J Clin Nutr 2004; 80: 114-22.
[42] Dubois MF, Hebert R. The incidence of vascular dementia in Canada: a comparison with Europe and East Asia. Neuroepidemiology 2001; 20: 179-187.

[43] Desai BS, Schneider JA, Li JL, Carvey PM, Hendey B. Evidence of angiogenic vessels in Alzheimer's disease. J Neural Transm 2009; 116: 587-97.

[44] Pirchl M, Ullrich C, Humpel C. Differential effects of short- and long-term hyperhomocysteinaemia on cholinergic neurons, spatial memory and microbleedings in vivo in rats. Eur J Neurosci 2010; 32: $1516-27$

[45] Herrmann W, Obeid R. Homocysteine: a biomarker in neurodegenerative diseases. Clin Chem Lab Med 2011; 49: 435-41.

[46] Humpel C. Chronic mild cerebrovascular dysfunction as a cause for Alzheimer's disease? Exp Gerontol 2011; 46: 225-32.

[47] Wehling M, Groth H. Challenges of longevity in developed countries: vascular prevention of dementia as an immediate clue to tackle an upcoming medical, social and economic stretch. Neurodegener Dis 2011; 8: 275-82.

[48] Agnati LF, Genedani S, Rasio G, et al. Studies on homocysteine plasma levels in Alzheimer's patients. Relevance for neurodegeneration. J Neural Transm 2005; 112: 163-69.

[49] Streck EL, Bavaresco CS, Netto CA, Wyse AT. Chronic hyperhomocysteinemia provokes a memory deficit in rats in the Morris water maze task. Behav Brain Res 2004; 153: 377-81.

[50] Hachinski V. Commentary on "Vascular cognitive impairment: today and tomorrow." Vascular cognitive impairment: yesterday, today, and tomorrow. Alzheimers Dement 2006; 2: 198-99.

[51] Breteler MM. Vascular risk factors for Alzheimer's disease: an epidemiologic perspective. Neurobiol Aging 2000; 21: 153-60.

[52] Troen A, Rosenberg. Homocysteine and cognitive function. Semin Vasc Med 2005; 2: 209-14.

[53] Kamat PK, Rai S, Swarnkar S, et al. Okadaic acid-induced Tau phosphorylation in rat brain: role of NMDA receptor. Neuroscience. 2013; 238: 97-13

[54] Hardy J, Selkoe DJ. The amyloid hypothesis of Alzheimer's disease: progress and problems on the road to therapeutics. Science 2002; 297: 353-56

[55] Isobe C, Abe T, Terayama Y. Homocysteine may contribute to pathogenesis of RNA damage in brains with Alzheimer's disease. Neurodegener Dis 2009; 6: 252-57.

[56] Tiraboschi P, Sabbagh MN, Hansen LA, et al. Alzheimer disease without neocortical neurofibrillary tangles: "a second look". Neurology 2004; 62: 1141-47.

[57] Rhodehouse BC1, Erickson MA, Banks WA, Bearden SE Hyperhomocysteinemic mice show cognitive impairment without features of Alzheimer's disease phenotype. J Alzheimers Dis 2013; 35: 59-66.

[58] Chai GS, Jiang X, Ni ZF, et al. Betaine attenuates Alzheimer-like pathological changes and memory deficits induced by homocysteine. J Neurochem 2013; 124: 388-96.

[59] Zhang CE, Wei W, Liu YH, et al. Hyperhomocysteinemia increases beta-amyloid by enhancing expression of gammasecretase and phosphorylation of amyloid precursor protein in rat brain. Am J Pathol 2009; 174: 1481-91.

[60] Kruman II, Kumaravel TS, Lohani A, et al. Folic acid deficiency and homocysteine impair DNA repair in hippocampal neurons and sensitize them to amyloid toxicity in experimental models of Alzheimer's disease. J Neurosci. 2002; 5: 1752-62.

[61] Koladiya RU, Jaggi AS, Singh N, Sharma BK. Ameliorative role of Atorvastatin and Pitavastatin in L-Methionine induced vascular dementia in rats. BMC Pharmacol 2008; 8: 14.

[62] Pacheco-Quinto J, Rodriguez de Turco EB, DeRosa S, et al. Hyperhomocysteinemic Alzheimer's mouse model of amyloidosis shows increased brain amyloid beta peptide levels. Neurobiol Dis 2006; 22: 651-56. 